\title{
Periprosthetic proximal medial femoral cortical destruction caused by a femoral arterial pseudoaneurysm
}

This article was published in the following Dove Press journal:

Clinical Interventions in Aging

17 December 2015

Number of times this article has been viewed

\author{
Uğur Tiftikçi \\ Sancar Serbest \\ Department of Orthopaedics and \\ Traumatology, Faculty of Medicine, \\ Kırıkkale University, Kırıkkale, Turkey
}

\begin{abstract}
A femoral artery aneurysm is a very rare complication of hip surgery treatment. In this presentation, we report a rare case of bone destruction caused by the femoral artery aneurysm after a hip hemiarthroplasty operation as a late stage complication. A 77-year-old male patient presented with swelling and pain in the hip without any signs of ischemia. X-ray showed a $7 \mathrm{~cm}$ bone destruction in the medial cortex of the proximal femur. After ultrasound imaging, a femoral artery aneurysm was diagnosed. The patient was operated on by vascular surgeons, and a large amount of organized hematoma with artery aneurysm was removed. The diagnosis of femoral aneurysm after hip surgery treatment can be difficult. Femoral aneurysm is rare and usually secondary to trauma, infection, or iatrogenic injury. We assumed that this case occurred during the hemiarthroplasty operation, caused by a compressing effect to the bone causing destruction. The patient was followed for 2 years, during which time the preoperative symptoms reduced dramatically and Harris Hip Score improved. After a hip arthroplasty operation, it must be kept in mind that a femoral arterial aneurysm can cause persistent symptoms. This case illustrates the importance of considering a pseudoaneurysm as a possible cause of periprosthetic osteolysis, particularly if rapid progressive growth has occurred. If this vascular injury had not been correctly diagnosed, the method of treatment could have been much different, probably with catastrophic consequences.
\end{abstract}

Keywords: femoral artery aneurysm, arthroplasty, bone, osteolysis

\section{Introduction}

Although vascular pathologies are less common in hip surgery, they can cause death or loss of extremities. Acute bleeding, arteriovenous fistula, thrombus formation, and pseudoaneurysms can develop as a result of vascular disorders occurring during surgeries in the hip region. ${ }^{1,2}$ The most common causes of the periprosthetic osteolysis are corrosion particles from implants, infection, metabolic disorders, and oncologic diseases. To the best of our knowledge, in existing literature there has only been one reported case in which periprosthetic osteolysis developed due to a pseudoaneurysm after prosthesis surgery. ${ }^{3}$ Here, we aim to add to the literature by presenting this patient as a second case. Written informed consent was obtained from the patient for publication of this Case report and any accompanying images. Institutional Review Board approval was not deemed necessary. All principles outlined in the Declaration of Helsinki were followed.

Correspondence: Sancar Serbest

Department of Orthopaedics and

Traumatology, Faculty of Medicine,

Kırıkkale University, Yahșiyan,

7II 100 Kırıkkale, Turkey

Tel +905335545080

Fax +903182252819

Email dr.sancarserbest@hotmail.com

\section{Case report}

A 77-year-old male presented at our clinic with hip pain on the left side. The patient had no comorbid disease other than hypertension. In the anamnesis, it was found that 
the patient had undergone hemiarthroplasty at another center because of a pertrochanteric fracture 2 years previously, and had no complaint after surgery. However, he began to experience hip pain in the 6 months before presenting at our clinic, with the pain becaming increasingly severe during the last 4 months in particular. During the physical examination, an incision scar was observed at the anterolateral region of the hip. The patient had no fever or local calor. The color of his skin was normal, but there was swelling at the thigh. The patient's range of motion was normal at the hip, but the movements were painful. The neuromuscular examination was normal, without any vascular pathology. On pelvis and hip radiographs, it was seen that bipolar hemiarthroplasty was performed on the hip, and there was a periprosthetic destructive area $(7 \mathrm{~cm}$ in size $)$ at the femoral medial cortex below the lesser trochanter (Figure 1). The following results were obtained in laboratory examination: white blood cell count was slightly elevated $\left(10.9 \times 10^{9} / \mathrm{L}\right)$, erythrocyte sedimentation rate (ESR) was $42 \mathrm{~mm} / \mathrm{h}$ (normal, 2-49 mm/h), and C-reactive protein (CRP) level was $45 \mathrm{mg} / \mathrm{L}$ (normal, 0-5 mg/L). Initially, the patient was considered to have osteolysis caused by metal particles. Minimal osteoblastic activity was detected via scintigraphy. Sonography was performed to rule out a mass, abscess, or infection as there was swelling at the hip. The sonography revealed an aneurysm, which was then confirmed by Doppler sonography. The patient underwent angiography and a pseudoaneurysm was detected in the profunda femoris (Figure 2).

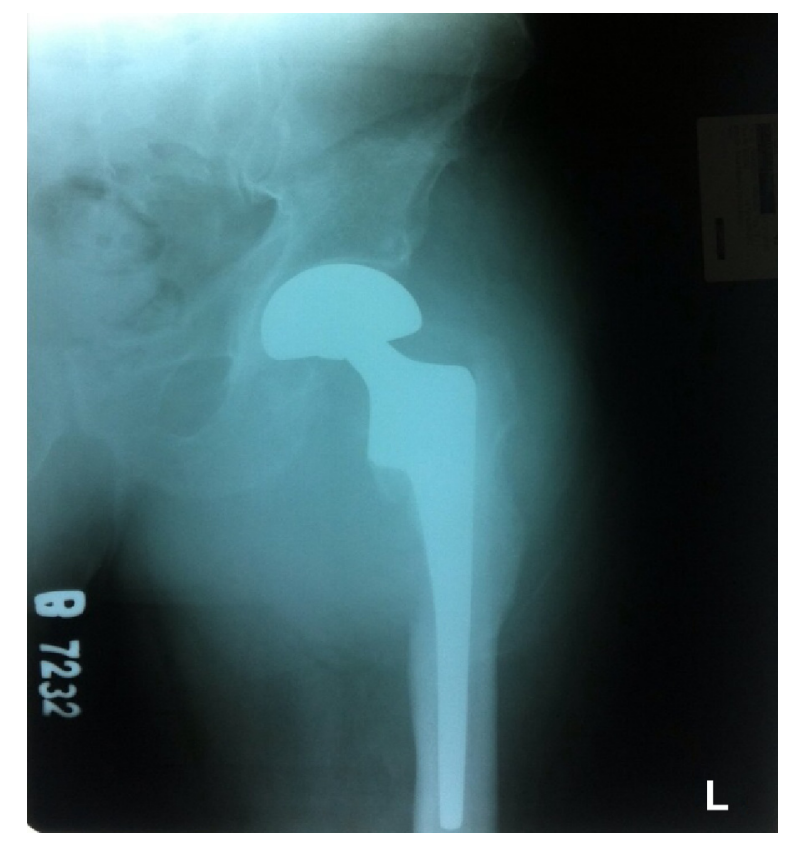

Figure I Radiographs showing the periprosthetic destructive area at femoral medial cortex below lesser trochanter.

Abbreviation: L, left.

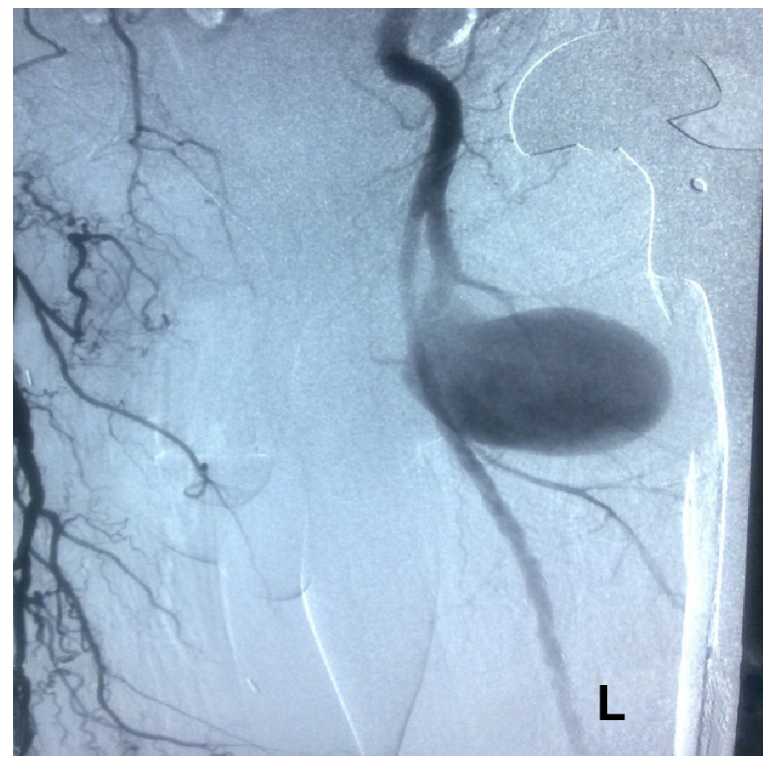

Figure 2 Angiogram showing the pseudoaneurysm was detected in profunda branch of femoris artery and periprosthetic destructive area. Abbreviation: L, left.

The patient's femoral artery and aneurysm were dissected and a thrombozed hematoma and fibrous tissue were removed via a long groin incision by a cardiovascular surgeon. Intraoperative culture tests were reported as negative. Specimens were reported as a nonspecific inflammatory reaction by the pathology department. The patient was mobilized on axillary crutches for 2 months. Hip pain declined rapidly in the postoperative period and it was found that ESR and CRP values were normalized. It was observed that remodeling was initiated on the osteolysis site in a radiograph obtained during the 2nd month after surgery (Figure 3 ). It was also

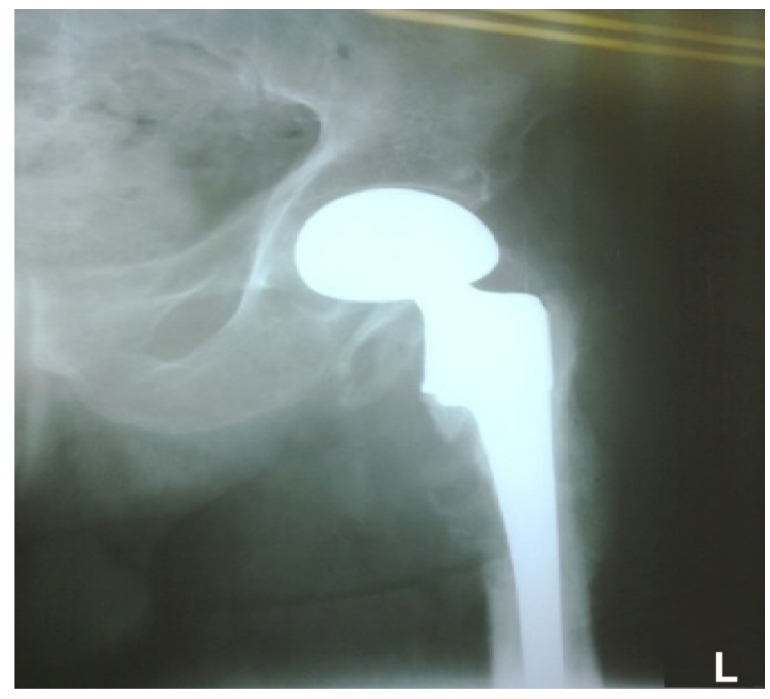

Figure 3 Radiographs showing the remodeling initiated in osteolysis site at month 2 after surgery.

Abbreviation: L, left. 


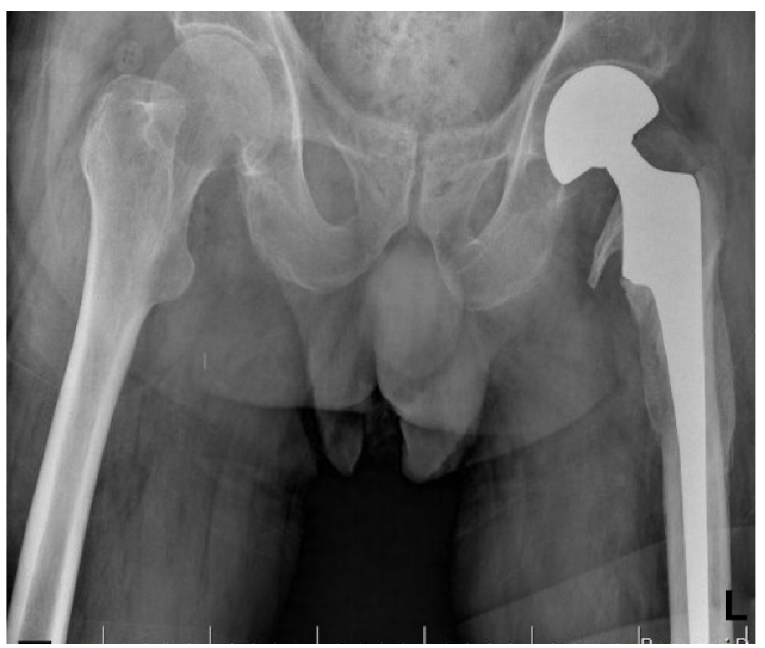

Figure 4 On the control visit at year 2, it was seen that the osteolytic area had completely recovered.

Abbreviation: L, left.

seen that the hip pain was completely resolved. The patient was allowed to walk without support. On the control visit in the 2nd year post-operation, it was seen that the osteolytic area had completely recovered, with a Harris Hip Score of 79.25 (Figure 4).

\section{Discussion}

Vascular injuries are rare complications during hip surgery, with reported prevalence of $0.25 \% .{ }^{1}$ However, in recent years, such injuries may have increased in prevalence due to an increased number of revision surgeries. Vascular complications are important as they may be life-threatening or cause loss of extremities. ${ }^{4}$ In a study by Shoenfeld et al, it was found that the mortality rate was $7 \%$ and the amputation rate was $15 \%$ in vascular injuries developed after total hip arthroplasty. ${ }^{5}$ In a study by Troutman et al, it was reported that the acute arterial injury was developed in 49 patients after knee and hip arthroplasty. ${ }^{6}$ Many studies have reported increasing prevalence of vascular disorders.

Direct injury is the most common mechanism of vascular injury. ${ }^{6}$ Factors involved in vascular injury include extreme reduction, distraction maneuvers, uncontrolled placement of Hoffmann retractors, acetabular drills, and migration of acetabular components, polymerization induced by high temperatures resulting from cauterization during surgery, thermal damage caused by cement leakage, and loss of elasticity due to arteriosclerosis..$^{7-10}$ In our case, the definitive mechanism of injury was unknown as the patient underwent surgery in another facility. However, we inferred two potential causes: laceration of a sclerotic vessel by a Hohmann retractor during an anterolateral approach and the thermal effect of cautery.
Pseudoaneurysm occurs when there is a small laceration of the wall of a large artery with continuing leakage of blood into the surrounding tissues. Clinically, it presents with discharge from the wound, internal bleeding, absent or weak distal pulses, and compartment syndrome if acute vascular injury is present. In minor injuries, the onset of clinical presentation is delayed, manifesting with swelling, pain, and anemia in general. ${ }^{11}$ In a study by Garrido-Gómez et al, it was suggested that severe bleeding may occur in the postoperative period. It was shown that small pseudoaneurysms can undergo spontaneous thrombosis within 4 weeks. ${ }^{12}$ However, the resolution cannot be predicted and thus a rupture can occur. During physical examination, pulsatile beat and occasionally arterial sound can be heard. In the hip, symptoms may be less severe, and chronic pain, mass effect, discharge, or local hematoma can develop in such patients. In our case, a pseudoaneurysm with delayed onset resulted in osteolysis by compressing the osseous tissue. Bone destruction was spontaneously resolved after eliminating the compressive effect of the mass.

Only one case with bone destruction osteolysis was reported in existing literature. In that case, osteolysis was detected in a hip radiograph obtained in the 9th month postoperation in addition to elevated ESR and CRP values in a patient who presented with subacute pain and minimal swelling. The case was initially considered as an infection. However, scintigraphy was inconclusive and the diagnosis was made by magnetic resonance imaging, which was then confirmed by angiography. No revision surgery was performed in that case. ${ }^{9}$ Similar clinical complaints and radiological findings were present in our case. The diagnosis was made more readily by sonographic evaluation in our case, which was then confirmed using angiography. Revision surgery was delayed due to the high risk of arthroplasty revision surgery together with cardiovascular surgeons, loss of extremity and mortality although osteolysis was more severe. The hip pain subsequently improved rapidly and revision surgery was not needed as the osteolysis was resolved by a callus.

According to existing literature, a pseudoaneurysm can be treated by direct saturation, repair using a patch and aneurysm resection; in recent years, minimally invasive coils have been used in the treatment of pseudoaneurysms developed after arthroplasty. ${ }^{7,9,13}$ In our study, aneurysm excision was preferred by the cardiovascular surgeon due to the extent of the aneurysm.

\section{Conclusion}

Vascular injuries following hip surgery can manifest with bleeding, compartment syndrome, anxmia, weak or 
absent filling of lower extremity vessels, and circulation abnormalities during the acute period, and with chronic pain, swelling, local mass effect, and weakness in the filling of the vessel in the late phase. One should be careful regarding vascular injuries during early and late postoperative periods since early diagnosis and appropriate management reduce morbidity and mortality. Adopting an alternate approach if vessels are calcified, such as using an anterior approach rather than a posterior approach for a hip replacement, may reduce the risk of vascular injuries. It should be kept in mind that osteolysis following arthroplasty can result from a pseudoaneurysm as a late complication, and that sonographic evaluation or angiography should be performed to minimize the risk of vascular injury before revision surgery.

\section{Disclosure}

The authors report no conflicts of interest in this work.

\section{References}

1. Nachbur B, Meyer RP, Verkkala K, Zürcher R. The mechanisms of severe arterial injury in surgery of the hip joint. Clin Orthop Relat Res. 1979;141:122-133.

2. Calligaro KD, Dougherty MJ, Ryan S, Booth RE. Acute arterial complications associated with total hip and knee arthroplasty. J Vasc Surg. 2003;38:1170-1177.
3. Harris WH. Wear and periprosthetic osteolysis the problem. Clin Orthop Relat Res. 2001;393:66-70.

4. Moreta J, Sáez de Ugarte O, Larruscain JF, Uriarte I, Jáuregui I, Martínez-De Los Mozos JL. Arterial pseudoaneurysm as a cause of periprosthetic osteolysis mimicking an infected total hip arthroplasty. J Orthop Sci. 2015;20(3):555-558. doi: 10.1007/s00776013-0488-9.

5. Shoenfeld NA, Stuchin SA, Pearl R, Haveson S. The management of vascular injuries associated with total hip arthroplasty. J Vasc Surg. 1990;11:549-555.

6. Troutman DA, Dougherty MJ, Spivack AI, Calligaro KD. Updated strategies to treat acute arterial complications associated with total knee and hip arthroplasty. J Vasc Surg. 2013;58(4):1037-1042.

7. Parvizi J, Pulido L, Slenker N, Macgibeny M, Purtill JJ, Rothman RH. Vascular injuries after total joint arthroplasty. J Arthroplasty. 2008; 23:1115-1121.

8. Mallory TH, Jaffe SL, Eberle RW. False aneurysm of the common femoral artery after total hip arthroplasty. A case report. Clin Orthop Relat Res. 1997;338:105-108.

9. Tkaczuk H. False aneurysm of the external iliac artery following hip endoprosthesis. Acta Orthop Scand. 1976;47:317-319.

10. Akizuki S, Terayama K, Kobayashi S. False aneurysm of the external iliac artery during total hip replacement. A case report. Arch Orthop Trauma Surg. 1984;102:210-211.

11. Rossi FH, Izukawa NM, et al. Effects of electrocautery to provoke endovascular thermal injury. Acta Cir Bras. 2011;26:329-332.

12. Garrido-Gómez J, Garrido-Gómez MN, Arrabal-Polo MA, Garrido-Pareja F, Linares-Palomino JP. Iatrogenic false aneurysms. A rare complication of hip surgery. Hip Int. 2012;22:397-402.

13. Ahmad F, Turner SA, Torrie P, Gibson M. Iatrogenic femoral artery pseudoaneurysms - a review of current methods of diagnosis and treatment. Clin Radiol. 2008;63:1310-1316.
Clinical Interventions in Aging

\section{Publish your work in this journal}

Clinical Interventions in Aging is an international, peer-reviewed journal focusing on evidence-based reports on the value or lack thereof of treatments intended to prevent or delay the onset of maladaptive correlates of aging in human beings. This journal is indexed on PubMed Central, MedLine,

\section{Dovepress}

CAS, Scopus and the Elsevier Bibliographic databases. The manuscript management system is completely online and includes a very quick and fair peer-review system, which is all easy to use. Visit http://www.dovepress. com/testimonials.php to read real quotes from published authors. 